\title{
Performance Analysis of Cognitive Radio Networks Adopting Packet Discarding or Buffering Policy
}

\author{
Min Zhang and Bin $\mathrm{Li}$ \\ School of Electronic and Information Engineering, South China University of \\ Technology, Guangzhou, 510640, China \\ E-mail:aries.zm@gmail.com
}

\begin{abstract}
In a cognitive radio network (CRN), a preempted secondary user (SU) is placed in a call level queue to wait for accessing another free channel. If the availability of channels is transparent to SUs, packets will be generated during their waiting time and the performance of the CRN will be influenced by which way to handle these packets. This paper mainly analyzes the performance of a CRN adopting discarding or buffering policy, Here, an analytical model is developed to derive the formulas for both call level performance measures (i.e., call blocking probability) and packet level performance measures (i.e., packet delay, packet loss ratio and throughput) for SUs. Numerical results show that theoretical models are consistent with simulation results. The major observations include (i) the performances of a CRN degrade as the primary user (PU) or $S U$ call arrival rate increases. (ii) Under discarding policy, the call blocking probability and packet delay are smaller than adopting buffering policy, while the packet delay and throughput are greater. (iii) Adopting different policies cause a smaller effect on call blocking probability and throughput than on packet loss ratio and packet delay.
\end{abstract}

Keywords: Cognitive radio network, call blocking probability, packet delay, packet loss ratio, throughput.

\section{Introduction}

With the rapid growth in demand for wireless communications, the spectrum resources become scarce. According to Federal Communications Commission (FCC), the unlicensed portions of the spectrum in which most wireless networks operate are heavily occupied, whereas the licensed portions of the spectrum are sporadically used [1]. In a $\mathrm{CRN}$, an unlicensed user (SU) opportunistically access a spectrum hole, which is a channel assigned to a licensed user (PU) but not being used at a particular time and geographic location. With the help of the cognitive radio technology, the SUs can access these spectrum holes without affecting the PUs [2]. The utilization of the radio spectrum can be improved significantly by employing the cognitive radio technology. However, this channel cannot be continuously utilized by the SU due to the presence of the PU. Thus, an SU must vacate the channel once detecting a PU appearance in it. At the same time, the SU will scan all the channels and handoff to another unused one if available; otherwise, it is preempted. A preempted SU can either leave the system or wait in a queue, and accordingly, its connection is terminated or suspended. The performance study of a CRN with such SUs' behaviors is important to understand the performance of the whole CRN.

There are some studies on the call level performance analysis of CRNs [3-11]. Zhu in [3] proposes a channel reservation scheme for a licensed spectrum sharing system and finds that forced termination probability can be greatly reduced through the reservation. In [4], a finite queue is introduced to store the newly arriving SU if there is no idle channel available, which is able to significantly reduce the SU call blocking probability and non- 
completion probability. The SUs are classified into two priority classes in [5]. A number of channels are reserved for the high priority SUs and the optimal reservation can be obtained. In [6], a mathematical analysis for CRN with imperfect sensing results is introduced. The call blocking probability, the termination probability and the spectrum utilization are analyzed. In addition, the performance of a CRN under realistic channel switching agility is studied in [7]. In [3]-[7], the SU is assumed to leave the system and terminate its connection once it is preempted. While in [8], Tang introduces a preemption queue for SUs to suspend their connections. A SU remains in the preemption queue until another channel is released. Expressions for the blocking probabilities and waiting times are derived. In [9], non-real-time calls are allowed to wait in buffers, while real-time calls are not allowed. The performance measures are derived in terms of service completion time, blocking probability and forced termination probability for both real-time and nonreal-time traffic. In [10], the state dependent access probabilities for SUs are optimized so that the spectrum resources can be efficiently and fairly shared by the SUs in an opportunistic way without interrupting the primary usage. Moreover, the preemption queue is able to reduce the SU blocking probability while increasing its throughput [11].

In all of the studies above, the packet level policies in a CRN are not considered. But according to our previous works [12], if the availability of channels is transparent to SUs, packets will be generated by them during their suspending period and the performance of a CRN will be influenced by which way to handle these packets. In this paper, we consider the SUs adopting discarding or buffering policy. Under the packet discarding policy, each SU discards the packets during their waiting time, and we call a CRN with such discarding policy as CRN-Discard. On the other hand, under the packet buffering policy, each SU just saves all the packets in a packet buffer during their waiting time, and we call a CRN with such buffering policy as CRN-buffer. Furthermore, the performances of a CRN against these two policies at both the call level and packet level are analyzed, and the formulas for call blocking probability, packet loss ratio, packet delay, and throughput for SUs are derived.

The remainder of the paper is organized as follows. Section 2 presents the system and traffic model. An analytical model for CRN-Discard is described in Section 3, while Section 4 presents the analytical model for CRN-Buffer. The numerical results are discussed in Section 5 and the paper is concluded in Section 6.

\section{System Model}

Consider a CRN with $N$ channels being shared by PUs and SUs. The call level model for a CRN is shown in Figure 1. The arrivals of PUs and SUs are modeled as Poisson processes with rates of $\lambda_{p}$ and $\lambda_{s}$, and the corresponding call duration is exponentially distributed with means of $1 / \mu_{p}$ and $1 / \mu_{s}$, respectively. We assume that one PU or SU requires one channel for service. For an SU call arrival, it is accepted only if there is a free channel. For a PU call arrival, since the existence of SUs is transparent to PUs, it is admitted if the number of PUs in service is less than $N$. When an accepted SU is preempted due to a PU arrival, the SU senses all the channels and handoff to an idle one if available; otherwise, it is placed in a queue to suspend its connection. The SU waits in the preemption queue until another channel is released by an SU or PU. 


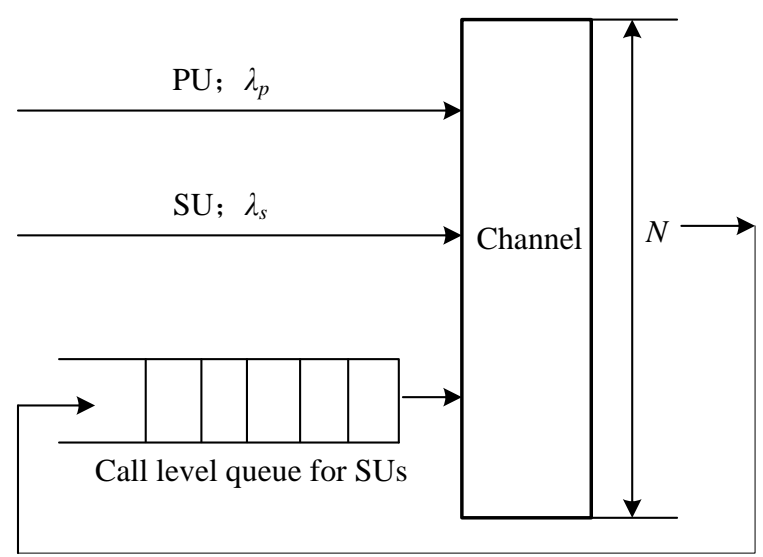

The preempted SUs

Figure 1. Call Level Model for a CRN

Packet flow generated by an SU

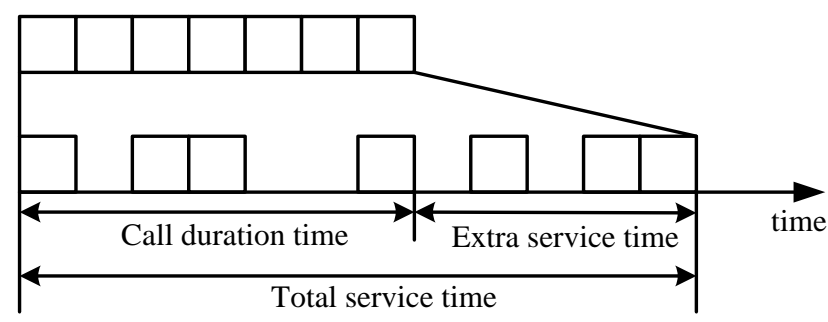

Figure 2. Packet Level Model for a CRN-Buffer

During SU call duration, one packet is generated in each time slot. The generated packet is sent if the SU gets an idle channel to transmit. While if the SU is waiting in the queue, the packet will be dropped or delayed in CRN-Discard or CRN-buffer, respectively. In the CRN-buffer, each SU has a packet buffer, where all the delayed packets are saved. At the end of the call duration, if the packet buffer is not empty, the service for the SU cannot end and the extra service time starts. One buffered packet can be sent if the SU accesses an idle channel in a time slot during the extra service time. As shown in Figure 2, the total service time is divided into two parts. In the first part, i.e., the call duration time, seven packets are generated by an SU, three of which are not transmitted successfully because the SU can not get an idle channel. In the second part, i.e., the extra service time, one buffered packet can be sent if the SU gets a channel to transmit in a time slot. The service cannot end until the call ends and the packet buffer become empty in a CRN-Buffer.

The spectrum sharing for SUs and PUs in a CRN can be modeled as a two-dimensional Markov model with states $(i, j)$. Let $P(i, j)$ be the stationary probability of the state $(i, j)$. The state space $\Gamma$ is given by

$$
\Gamma=\{(i, j) / 0 \leq i \leq N ; 0 \leq j \leq N\}
$$

In order to simplify the expressions of a formula, we define a function $\phi(i, j)$ as

$$
\phi(i, j)= \begin{cases}1, & (i, j) \in \Gamma \\ 0, & \text { otherwise }\end{cases}
$$

\section{Performance Analysis in CRN-Discard}


Since a PU accesses to the system as there is no SU, the PU's arrival and departure rate is $\lambda_{p}$ and $\mu_{p}$, respectively. When the total number of users in the system is smaller than $N$, a new arrival SU is admitted, and its arrival rate is $\lambda_{s}$; otherwise, a new arrival SU is blocked, and its arrival rate is zero. When an SU is in communication or waiting state in a timeslot, one packet is sent or discarded, respectively. The SU's service ends as the call ends and its departure rate is $\mu_{s}$. Figure 3 shows the state transition diagram of a CRNDiscard.

The steady-state balance equations for a CRN-Discard are listed below.

Case1) If $i+j<N, 0 \leq i \leq N$ and $0 \leq j \leq N$, then

$$
\begin{aligned}
& \left(i \mu_{p}+j \mu_{s}+\lambda_{p}+\lambda_{s}\right) P(i, j) \phi(i, j) \\
& =\lambda_{p} P(i-1, j) \phi(i-1, j)+\lambda_{s} P(i, j-1) \phi(i, j-1) \\
& +(i+1) \mu_{p} P(i+1, j) \phi(i+1, j)+(j+1) \mu_{s} P(i, j+1) \phi(i, j+1)
\end{aligned}
$$

Case2) If $i+j=N, 0 \leq i \leq N$ and $0 \leq j \leq N$, then

$$
\begin{aligned}
& {\left[i \mu_{p}+j \mu_{s}+\lambda_{p}\right] P(i, j) \phi(i, j)} \\
& =\lambda_{p} P(i-1, j) \phi(i-1, j)+\lambda_{s} P(i, j-1) \phi(i, j-1) \\
& +(i+1) \mu_{p} P(i+1, j) \phi(i+1, j)+j \mu_{s} P(i, j+1) \phi(i, j+1)
\end{aligned}
$$

Case3) If $i+j>N, 0 \leq i \leq N$ and $0 \leq j \leq N$, then

$$
\begin{aligned}
& {\left[i \mu_{p}+j \mu_{s}+\lambda_{p}\right] P(i, j) \phi(i, j)} \\
& =\lambda_{p} P(i-1, j) \phi(i-1, j)+(i+1) \mu_{p} P(i+1, j) \phi(i+1, j) \\
& +j \mu_{s} P(i, j+1) \phi(i, j+1)
\end{aligned}
$$

Where $P(i, j)$ satisfies the normalization constraint

$$
\sum_{\{(i, j) \in \Gamma\}} P(i, j)=1
$$

An SU's call blocking probability is the probability that the total number of users is greater than or equal to $N$, so the SU's call blocking probability $P_{b}$ can be expressed as

$$
P_{b}=\sum_{\{(i, j) \in \Gamma \mid i+j \geq N\}} P(i, j)
$$

An SU's waiting probability is the ratio of the number of preempted SUs to that of admitted SUs, that is,

$$
P_{w}=\frac{\sum_{\{(i, j) \in \Gamma[i+j>N\}}(i+j-N) P(i, j)}{\sum_{\{(i, j) \in \Gamma]} j P(i, j)}
$$

Because a SU discards packets in waiting state, so the probability that the SU discards packets is equal to its waiting probability. The packet loss ratio is,

$$
P_{l}=P_{w}=\frac{\sum_{\{(i, j) \in \Gamma[i+j>N\}}(i+j-N) P(i, j)}{\sum_{\{(i, j) \in \Gamma\}} j P(i, j)}
$$



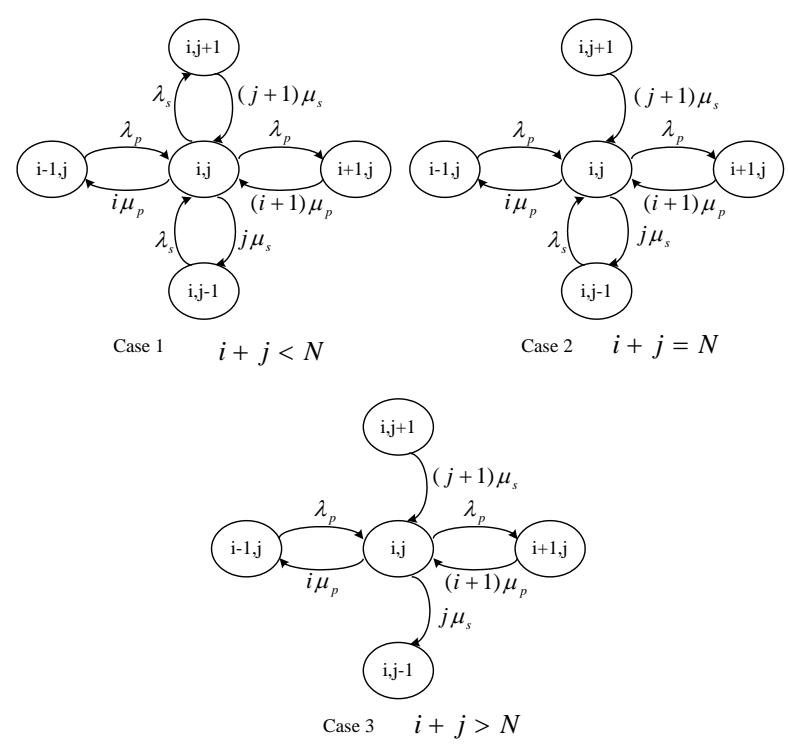

\section{Figure 3. State Transition Diagram of a CRN-Discard}

The throughput for SUs is defined as the total number of packets sent by all the successfully completed SU calls in a time slot [13]. Let $\bar{n}_{c}$ and $\bar{n}_{p}$ be the average number of completed SU calls per time slot and successfully transmitted packets per SU, respectively. The throughput can be given by the product of $\bar{n}_{c}$ and $\bar{n}_{p}$. Since the length of a time slot is much smaller than a call, the number of admitted and completed SUs in a time slot can be given by the admitted and completed rate, respectively. As we known as the queue theory [14], the admitted rate is the same as the completed rate when the system works in a steady state. So the number of completed SUs in a time slot is

$$
\bar{n}_{c}=\left(1-P_{b}\right) \lambda_{s}
$$

We know that the mean length of an SU packet flow is $\mu_{s}^{-1}$, so the number of successfully transmitted packets per SU is

$$
\bar{n}_{p}=\left(1-P_{l}\right) \mu_{s}^{-1}
$$

From Equations (10) and (11), the SU throughput can be expressed as

$$
\sigma_{s}=\bar{n}_{c} * \bar{n}_{p}=\left(1-P_{b}\right)\left(1-P_{l}\right) \frac{\lambda_{s}}{\mu_{s}}
$$

\section{Performance Analysis in CRN-Buffer}

Since a PU accesses a channel as there is no SU, the PU's arrival rate and departure rate is $\lambda_{p}$ and $\mu_{p}$, respectively. When the total number of users in the system is smaller than $N$, a new arrival SU is admitted, and its arrival rate is $\lambda_{s}$; otherwise, a new arrival SU is

blocked,

and

its 


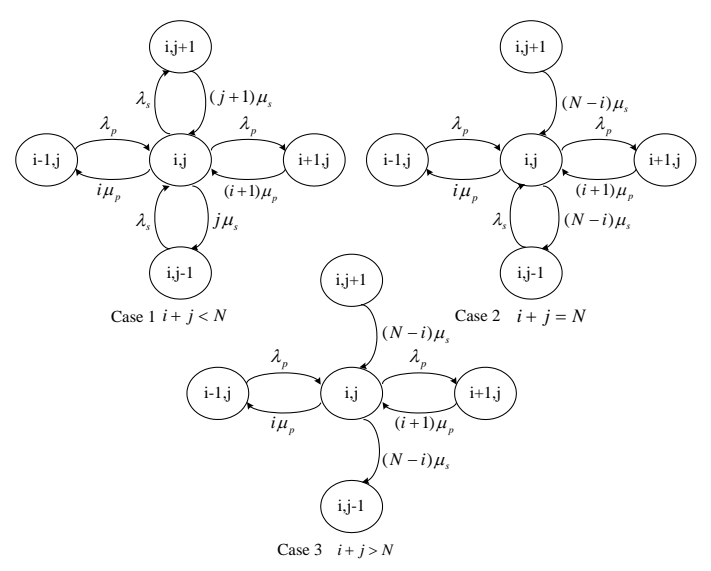

Figure 4. State Transition Diagram of a CRN-Buffer

Service ends as the call ends and its departure rate is $\mu_{s}$. When an SU is in waiting state, no packet can be sent and its service can not end, so the departure rate is zero. Figure 4 shows the state transition diagram of a CRN-Buffer. The steady-state balance equations for a CRN-Buffer are listed below.

Case1) If $i+j<N, 0 \leq i \leq N$ and $0 \leq j \leq N$, then

$$
\begin{aligned}
& \left(i \mu_{p}+j \mu_{s}+\lambda_{p}+\lambda_{s}\right) P(i, j) \phi(i, j) \\
& =\lambda_{p} P(i-1, j) \phi(i-1, j)+\lambda_{s} P(i, j-1) \phi(i, j-1) \\
& +(i+1) \mu_{p} P(i+1, j) \phi(i+1, j)+(j+1) \mu_{s} P(i, j+1) \phi(i, j+1)
\end{aligned}
$$

Case2) If $i+j=N, 0 \leq i \leq N$ and $0 \leq j \leq N$, then

$$
\begin{aligned}
& {\left[i \mu_{p}+(N-i) \mu_{s}+\lambda_{p}\right] P(i, j) \phi(i, j)} \\
& =\lambda_{p} P(i-1, j) \phi(i-1, j)+\lambda_{s} P(i, j-1) \phi(i, j-1) \\
& +(i+1) \mu_{p} P(i+1, j) \phi(i+1, j)+(N-i) \mu_{s} P(i, j+1) \phi(i, j+1)
\end{aligned}
$$

Case3) If $i+j>N, 0 \leq i \leq N$ and $0 \leq j \leq N$, then

$$
\begin{aligned}
& {\left[i \mu_{p}+(N-i) \mu_{s}+\lambda_{p}\right] P(i, j) \phi(i, j)} \\
& =\lambda_{p} P(i-1, j) \phi(i-1, j)+(i+1) \mu_{p} P(i+1, j) \phi(i+1, j) \\
& +(N-i) \mu_{s} P(i, j+1) \phi(i, j+1)
\end{aligned}
$$

Where $P(i, j)$ satisfies the same normalization constraint shown in equation (6).

An SU's call blocking probability and waiting probability in CRN-Buffer is the same as $\mathrm{CRN}$-discard, which is show in equation 7 and 8 , respectively.

In the CRN-buffer, an SU's delayed packets are saved in its packet buffer. Let the buffer length $x(i)$ be the amount of the packets saved in the buffer in a certain timeslot $i$. The total buffer length $\xi_{i}^{j}$ is defined as the sum of the buffer length from timeslot $i$ to $j$. Assuming that the length of an SU call duration is $n$ ( $n>0$ is an integer), and END (END $\geq n$ is a random variable) is the end of the total service time for the SU. From timeslot 1 to $n$, one packet is generated at every timeslot and delayed with a probability $P_{w}$. If a packet is delayed in time slot $i$, it increases the total buffer length during the call duration by $n-i+1$. Thus $\xi_{1}^{n}$ is

$$
\xi_{1}^{n}=\sum_{i=1}^{n} x(i)=\sum_{i=1}^{n}(n-i+1) P_{w}=\frac{n(n+1)}{2} P_{w}
$$


The extra service time lasts from timeslot $n+1$ to END, and one buffered packet can be sent with a probability $1-P_{w}$ in each timeslot. $\xi_{i}^{\mathrm{END}}(n<i<\mathrm{END})$ depends only on $x(i-1)$. Suppose $x(i-1)$ is $k$ and $\xi_{i}^{\mathrm{END}}$ is $\xi(k)$. In timeslot $i$, if the SU is preempted, $x(i)$ is still $k$ and $\xi_{i+1}^{\mathrm{END}}$ is $\xi(k)$; If not, $x(i)$ decreases to $k-1$ and $\xi_{i+1}^{\mathrm{END}}$ becomes to $\xi(k-1)$. Hence the relationship between $\xi(k)$ and $\xi(k-1)$ can be given by

$$
\xi(k)=[\xi(k)+k] P_{w}+[\xi(k-1)+k-1]\left(1-P_{w}\right)
$$

and $\xi(k)$ can be derived as

$$
\begin{aligned}
\xi(k) & =\frac{k}{1-P_{w}}-1+\xi(k-1) \\
& =\left(\frac{k}{1-P_{w}}-1\right)+\mathrm{L}+\left(\frac{2}{1-P_{w}}-1\right)+\xi(1) \\
& =\frac{k^{2}+k\left(2 P_{w}-1\right)-2 P_{w}}{2\left(1-P_{w}\right)}+\sum_{i=1}^{\infty} i P_{w}^{i}\left(1-P_{w}\right) \\
& =\frac{k^{2}+k\left(2 P_{w}-1\right)}{2\left(1-P_{w}\right)}
\end{aligned}
$$

Since the probability that $x(n)$ is $k$ means that there are $k$ packets delayed during the call duration, that is

$$
P[x(n)=k]=C_{n}^{k} P_{w}^{k}\left(1-P_{w}\right)^{n-k}
$$

The total buffer length in the extra service time is

$$
\begin{aligned}
& \xi_{n+1}^{\mathrm{END}}=\sum_{k=1}^{n} \xi(k) P[x(n)=k] \\
& =\sum_{k=1}^{n} C_{n}^{k} P_{w}{ }^{k}\left(1-P_{w}\right)^{n-k}\left\{\frac{k^{2}}{2\left(1-P_{w}\right)}+\frac{k\left[1-2\left(1-P_{w}\right)\right]}{2\left(1-P_{w}\right)}\right\} \\
& =\frac{n(n+1) P_{w}{ }^{2}}{2\left(1-P_{w}\right)}
\end{aligned}
$$

From Equations (16) and (20), the total buffer length within the SU's total service time is

$$
\xi_{1}^{\mathrm{END}}=\xi_{1}^{n}+\xi_{n+1}^{\mathrm{END}}=\frac{n(n+1)}{2} P_{w}+\frac{n(n+1) P_{w}{ }^{2}}{2\left(1-P_{w}\right)}=\frac{n(n+1) P_{w}}{2\left(1-P_{w}\right)}
$$

Because the call duration is exponentially distributed with means of $1 / \mu_{s}$, the probability that the length of an SU call is $n$ is

$$
P_{n}=\int_{n-0.5}^{n+0.5} \mu_{s} \exp \left(-\mu_{s} t\right) d t=\exp \left[-(n-0.5) \mu_{s}\right]-\exp \left[-(n+0.5) \mu_{s}\right]
$$

Therefore, the mean of $\xi_{1}^{\mathrm{END}}$ is

$$
\begin{aligned}
& \xi=\sum_{n=1}^{\infty} \xi_{1}^{\mathrm{END}} P_{n} \\
& =\sum_{n=1}^{\infty}\left\{\exp \left[-(n-0.5) \mu_{s}\right]-\exp \left[-(n+0.5) \mu_{s}\right]\right\} \frac{n(n+1) P_{w}}{2\left(1-P_{w}\right)} \\
& =\frac{\exp \left(-0.5 \mu_{s}\right) P_{w}}{\left[1-\exp \left(-\mu_{s}\right)\right]^{2}\left(1-P_{w}\right)}
\end{aligned}
$$


The average length of a packet flow generated during an SU's service time is $1 / \mu_{s}$, so the SU's packet delay can be calculated according to Little Theory [14] as follows

$$
D=\frac{\xi / \mathrm{END}}{1 /\left(\mu_{s} \mathrm{END}\right)}=\xi \mu_{s}=\frac{\mu_{s} \exp \left(-0.5 \mu_{s}\right) P_{w}}{\left[1-\exp \left(-\mu_{s}\right)\right]^{2}\left(1-P_{w}\right)}
$$

The SU throughput can be expressed as the same as equation (15), that is,

$$
\sigma_{s}=\left(1-P_{s}\right)\left(1-P_{l}\right) \frac{\lambda_{s}}{\mu_{s}}=\left(1-P_{s}\right) \frac{\lambda_{s}}{\mu_{s}}
$$

\section{Numerical Results}

The proposed system models for CRN-Discard and CRN-buffer are simulated in MATLAB. To evaluate the accuracy of the analytical model, we compare the results obtained from the analytical model and the simulation. The parameters are set as follows: $N=11, \mu_{p}=0.01$ and $\mu_{s}=0.02$. The abbreviations for CRN-Discard and CRN-Buffer are C-D and $\mathrm{C}-\mathrm{B}$, respectively. Symbol $\mathrm{T}$ and $\mathrm{S}$ in the figures indicate theoretical and simulation results, respectively. Figures 5 6 show the simulated and analytical results for SU call blocking probability, packet loss ratio and packet delay of a CRN varying with PU and SU call arrival rates. It can be seen that the proposed analytical models fit the simulation results very well. It also can be seen from the figures that adopting different packet level policies cause a smaller effect on call blocking probability and throughput than on packet loss ratio and packet delay.

Figure 5(a) shows the simulated call blocking probability and the theoretical results for the CRN-Discard and CRN-Buffer. As the call arrival rate $\lambda_{p}$ or $\lambda_{s}$ increases, less channels are available and more SUs contend for the available channels, which causes an increase in SU call blocking probability. Additionally, the call blocking probability in CRN-Buffer is a little greater than in CRN-Discard. This is because, under the packet buffering policy, the traffic load is more heavily in CRN-buffer than that in CRN-Discard.

Figure 5(b) shows the simulated packet loss ratio and the theoretical results for the CRN-Discard. As the call arrival rate $\lambda_{p}$ or $\lambda_{s}$ increases, the packet loss ratio in CRNDiscard increases. It is obvious that the packet loss ratio in CRN-Buffer is always zero. While under the packet discarding policy, an increase in $\lambda_{p}$ or $\lambda_{s}$ causes a higher waiting probability, which leads to more discarded packets.

Figure 6(a) depicts the simulated packet delay and the theoretical results for the CRNBuffer. As the call arrival rate $\lambda_{p}$ or $\lambda_{s}$ increases, the packet delay in CRN-Buffer increases. It is obvious that the packet delay in CRN-Discard is always zero. While under the packet buffering policy, an increase in $\lambda_{p}$ or $\lambda_{s}$ causes a higher waiting probability, which leads to longer waiting time and more discarded packet.

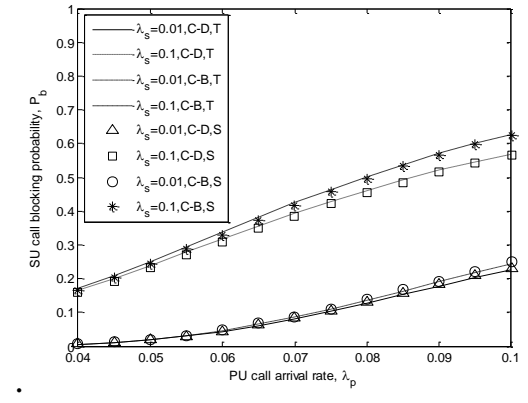

(a) SU Call Blocking Probability

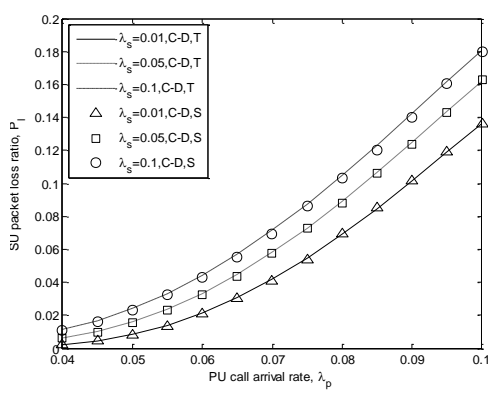

(b) SU Packet Loss Ratio

Figure 5. SU Call Blocking Probability and Packet Loss Ratio Relative to the Call Arrival Rates of PUs and SUs 


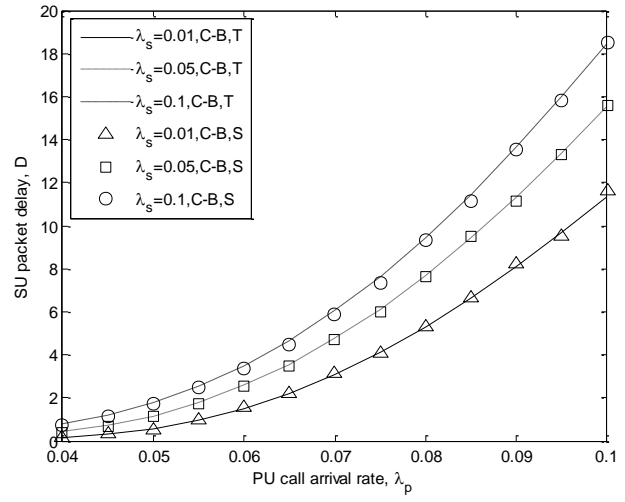

(a) SU packet delay

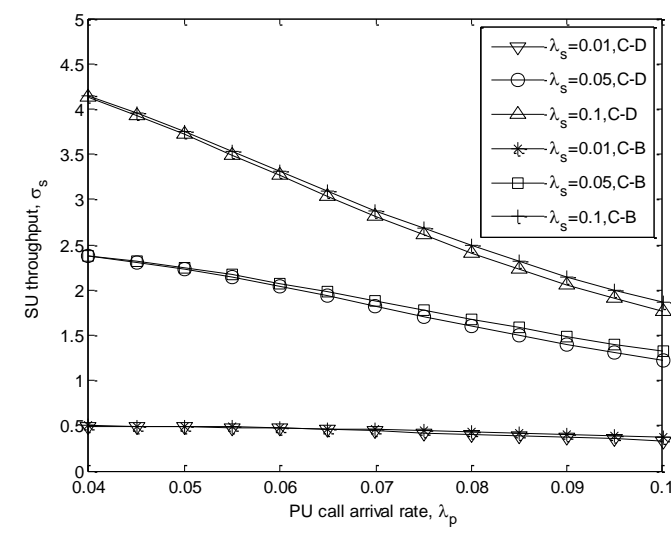

(b) SU throughput

Figure 6. SU Packet Delay and Throughput Relative to the Call Arrival Rates of PUs and SUs

Figure 6(b) shows the theoretical results of throughput for the CRN-Discard and CRNBuffer. As the call arrival rate $\lambda_{p}$ or $\lambda_{s}$ increases, less SUs are accepted in the system and more packets are discarded, which causes a decrease in SU throughput. Additionally, the difference of throughput in CRN-Buffer and CRN-Discard is small.

\section{Conclusion}

In this paper, the preempted SUs are suspended to wait for another channel to avoid direct leaving. Packets generated during the waiting time are discarded and saved in CRN-Discard and CRN-buffer, respectively. Analytical models are developed to study the call level and packet level performances of a CRN, which provides an understanding of the connection between the performances of these two levels. The formulas for the call blocking probability, packet loss ratio, packet delay and throughput for SUs are derived. Numerical results show that the proposed analytical model fits the simulation results very well. The performances of a CRN degrade as the PU or SU call arrival rate increases. The call blocking probability and packet delay in CRN-Discard are smaller than in CRNBuffer, while the packet delay and throughput are greater. Different policies can not make much difference to call blocking probability and throughput, but has a large effect on packet loss ratio and packet delay.

\section{Acknowledgements}

This work was supported partially by the National Natural Science Foundation of People's Republic of China (61301300 and 61302058).

\section{References}

[1] "Spectrum policy task force", Federal Commun. Commiss., Washington, DC, Rep. OET Docket (2003), pp. 02-135.

[2] S. Haykin, "Cognitive radio: brain-empowered wireless communications", IEEE Journal on Selected Areas in Commun (JSAC), vol. 23, no.2, (2005), pp.201-220.

[3] X. Zhu, L. Shen, and T.-S. P. Yum, “Analysis of cognitive radio spectrum access with optimal channel reservation", IEEE Commun. Letters, vol. 11, no.4, (2007), pp.304-306.

[4] Y. Zhang, "Dynamic spectrum access in cognitive radio wireless networks", IEEE ICC 2008, (2008), pp. 4927-4932.

[5] V. K. Tumuluru, P. Wang and D. Niyato, "Performance analysis of cognitive radio spectrum access with prioritized traffic", IEEE ICC, (2011), pp.1-5. 
[6] O. Altrad, S. Muhaidat, A. Al-Dweik, "Opportunistic spectrum access in cognitive radio networks under imperfect spectrum sensing", IEEE Transactions on Vehicular Technology, Vol. 63, no. 2, (2014), pp. 920-925.

[7] M. NoroozOliaee, B. Hamdaoui and X. Cheng, "Analyzing cognitive network access efficiency under limited spectrum handoff agility", IEEE Transactions on Vehicular Technology, Vol. 63, no. 3, (2014), pp. 1402-1407.

[8] S. Tang and B. L. Mark, "Analysis of opportunistic spectrum sharing with markovian arrivals and phase-type service", IEEE Transactions on Wireless Communications, vol. 8, no. 6, (2009), pp. 31423150.

[9] J. Heo, J. Shin and J. Nam, "Mathematical analysis of secondary user traffic in cognitive radio system", 68th IEEE International Conference on Vehicular Technology Conference, Calgary (2008).

[10] B. Wang, J. Zhu and K. Liu., "Primary-prioritized Markov approach for dynamic spectrum allocation", IEEE Transactions on Wireless Communications, vol. 8, no. 4, (2009), pp.1854-1865.

[11] T. M. N. Ngatched, S. Dong and A. S. Alfa, "Analysis of cognitive radio networks with channel assembling, buffering, and imperfect sensing”, IEEE WCNC, Shanghai (2013), pp.952-957.

[12] M. Zhang, S. M. Jiang, G. Wei and H. J. Wang, "Performance anaylisis of cognitive radio networks against secondary user's policies", the 8th IEEE/ACIS Int. Conf. on Computer and Information Science, Shanghai (2009).

[13] W. Ahmed, J. Gao and H. Suraweera, "Comments on "analysis of cognitive radio spectrum access with optimal channel reservation", IEEE Transactions on Wireless Communications, vol. 8, no. 9, (2014), pp. 4488-4491.

[14] D. Kannan, “An Introduction to Stochastic Processes. Elservier North Holland Inc”, (1979).

\section{Authors}

Min Zhang, she is a postdoctoral student at South China University of Technology, Guangzhou, China. She received the Ph.D. in Telecommunication and information system from South China University of Technology in 2010. Her research interests include resource allocation and optimization for cognitive radio networks.

Bin Li, she received the B.S. and M.S. degrees in electrical engineering from the South China University of Technology, Guangzhou, China, in 1989 and 1992, respectively, and the Ph.D. degree in electrical engineering from the University of Hong Kong, Hong Kong, in 2001. She is currently a professor in the Institute of Microelectronics at the South China University of Technology. 